\title{
EFEITO DA TEMPERATURA E DO USO DE EMBALAGEM NA CONSERVAÇÃO PÓS-COLHEITA DE FRUTOS DE CAGAITA (Eugenia dysenterica DC. $)^{1}$
}

\author{
JULIANA DE OLIVEIRA CARNEIRO², MARIA APARECIDA DE ALENCAR DE SOUZA³, \\ YNAYARA JOANE DE MELO RODRIGUES ${ }^{3} \&$ ANA MARIA MAPELI $^{4}$
}

RESUMO-Eugenia dysenterica DC. (cagaiteira) destaca-se entre as espécies nativas do Cerrado por produzir frutos de sabor agradável, os quais podem ser consumidos tanto in natura quanto processados na forma de doces, compotas e geleias. Apesar do potencial econômico, é uma planta pouco explorada, principalmente devido à baixa durabilidade dos frutos. Assim, este trabalho teve como objetivo avaliar o efeito da embalagem e da temperatura sobre a conservação pós-colheita de frutos de E. dysenterica. Para isto, os frutos de cagaita foram coletados no estádio verde-maduro, ainda ligados à planta-mãe, e levados ao Laboratório de Botânica da Universidade Federal da Bahia, onde foram selecionados quanto à integridade física, ausência de danos mecânicos e patogênicos. Após lavagem em água corrente, os frutos foram secos e acondicionados em bandejas de poliestireno expandido, cobertas por filme de policloreto de vinila (PVC) de 10 micras, perfurados e sem perfuração, e em bandejas sem revestimento de PVC. A perfuração foi realizada visando a maior circulação de ar dentro das embalagens. Em seguida, foram armazenados em duas temperaturas, 5 e $25^{\circ} \mathrm{C}$. Para a avaliação da durabilidade dos frutos, foram realizadas avaliações diárias das características físicas e químicas, incluindo coloração, firmeza, pH, perda de massa, altura e diâmetro. O metabolismo de carboidratos também foi avaliado por meio da quantificação dos açúcares solúveis. Os frutos da cagaita apresentaram durabilidade de 5 dias, independentemente dos tratamentos utilizados, sendo que os submetidos à refrigeração apresentaram sintomas de injúria por frio, alteração da coloração e firmeza ( $25 \%$ ), redução de $\mathrm{pH}$ e do consumo de carboidratos. Já em frutos mantidos a $25^{\circ} \mathrm{C}$, houve amarelecimento completo, perda de firmeza, aumento do $\mathrm{pH}$ e maior consumo de carboidratos. Verificou-se que o uso de embalagens, praticamente, não promoveu efeitos benéficos significativos. Desta forma, concluiu-se que o armazenamento dos frutos em temperatura de 25 e $5^{\circ} \mathrm{C}$ não é aconselhável, sendo que esta última causa injúrias, e os frutos, por serem climatéricos, têm vida de prateleira curta.

Termos para indexação: cagaita, armazenamento, cloreto de polivinila, vida de prateleira.

\section{EFFECT OF TEMPERATURE AND THE USE OF PACKING IN THE POST HARVEST CONSERVATION OF Eugenia dysenterica DC. FRUITS}

\begin{abstract}
Eugenia dysenterica DC. (cagaiteira) stands for being one of the native species from Cerrado that produces fruit of pleasant taste, which can be consumed both in natura and processed as sweets, jams and jellies. Despite its economic potential, it is a little explored plant due to the low durability of the fruits. So, this study aimed at evaluating the effect of packing and temperature upon the post harvest conservation of $E$. dysenterica fruits. In order to achieve it, the fruits of cagaita were collected in the mature green stage and still linked to the stock plant and taken to the Botanical Laboratory of the Federal University of Bahia, where they were selected according to their physical integrity, lack of mechanical and pathogenic damages. After being washed in running water, the fruits were dried and packed in trays made of expanded polystyrene and covered by perforated and non-perforated polyvinyl chloride film (PVC) of 10 microns and in trays without PVC. After that, they were stored in two temperatures, $5^{\circ}$ and $25^{\circ}$. Drilling was carried for increased air circulation inside the package. In order to evaluate the durability of the fruits, daily evaluations of physical and chemical characteristics were taken, including $\mathrm{pH}$, weight loss, height, diameter, hardness and coloring. The carbohydrate metabolism was also evaluated through the quantification of soluble sugars. The fruits of cagaita showed durability of 5 days, independent from the treatments used. The ones which were submitted to refrigeration showed symptoms of damage by cold, alteration of coloring and hardness $(25 \%)$, reduction of both $\mathrm{pH}$ and carbohydrates consumption. On the other hand, the fruits which were kept in $25^{\circ} \mathrm{C}$ suffered total yellowing, loss of hardness, increase of $\mathrm{pH}$ and higher carbohydrate consumption. It has been verified that the use of packing has practically not promoted significant beneficial effects. Thus, it can be concluded that the storage of the fruits in a temperature of 25 and $5^{\circ} \mathrm{C}$ are not advisable, and the last causes damages and, for being climacteric, the fruits have short shelf life.
\end{abstract}

Index terms: cagaita; storage; polyvinyl chloride; shelf life.

${ }^{1}$ (Trabalho 157-14). Recebido em: 23-04-2014. Aceito para publicação em: 09-04-2015. Parte da Dissertação de Mestrado da primeira autora. ${ }^{2}$ Mestra em Ciências Ambientais - Univ. Fed. da Bahia e Docente do Inst. Fed. da Bahia, Câmpus Barreiras. E-mail:julycarneiro@homail.com ${ }^{3}$ Discentes da Universidade Federal da Bahia. E-mail:maria88_alencar@hotmail.com, ynayarajoane@hotmail.com

${ }^{4}$ Orientadora, Docente da Universidade Federal da Bahia. E-mail: anammapeli@gmail.com 


\section{INTRODUÇÃO}

A flora do Cerrado possui diversas espécies frutíferas com grande potencial de utilização agrícola, que são tradicionalmente utilizadas pela população local, pois os frutos apresentam sabores sui generis e podem ser consumidos in natura ou na forma de sucos, licores, sorvetes, geleias e doces diversos (SILVA et al., 2008). Cardoso et al. (2011), ao caracterizarem física e quimicamente a polpa de cagaita coletada no Cerrado de Minas Gerais, observaram que os frutos apresentaram forma arredondada, casca frágil, alto rendimento de polpa, alta umidade e baixo valor energético, além de serem considerados fonte de vitaminas A e C.

Dentre estas espécies, destaca-se Eugenia dysenterica DC. (Myrtaceae), popularmente conhecida como cagaita, a qual possui fruto com formato globoso, bagáceo, cor amarelo-clara, levemente ácido, epicarpo membranoso, com peso entre 14 e $20 \mathrm{~g}$, comprimento de 3 a $4 \mathrm{~cm}$ e diâmetro de 3 a $5 \mathrm{~cm}$ (SILVA et al., 2001). Entretanto, a consistência de sua polpa dificulta a conservação pós-colheita dos frutos (NAVES et al., 2002), reduzindo seu período de comercialização; haja vista que os frutos são produtos perecíveis, pois apresentam atividade metabólica elevada, notadamente após a colheita, conduzindo-os aos processos de deterioração.

Após a retirada do fruto da planta-mãe, inicia-se a fase conhecida como pós-colheita, que prossegue até a sua utilização no preparo de alimentos para consumo ou processamento (RAHMAN, 2003). Este período é marcado pelos processos de transpiração, respiração e produção de etileno, principais fatores de deterioração que afetam significantemente a qualidade pós-colheita dos frutos e que estão relacionados ao fenômeno de senescência (ÁLVAREZ-ARMENTA et al., 2008).

O conhecimento da fisiologia póscolheita do fruto contribuirá para a elaboração de subsídios técnicos que visem a ampliar o tempo de armazenamento sem alterar as características físicas, organolépticas e nutricionais (ANTUNES et al., 2003).

A redução da temperatura e o aumento da umidade relativa são alguns dos principais parâmetros para manter a qualidade dos frutos durante o armazenamento e o transporte. A baixa temperatura diminui as atividades fisiológicas, bioquímicas e microbiológicas que ocasionam a deterioração do produto e a alteração de suas características, incluindo textura, sabor, cor e valor nutritivo, comprometendo a qualidade (SAMIRA et al., 2011). Outra alternativa para conservação de produtos hortícolas é a utilização de embalagens plásticas, pois mantém alta a umidade relativa ao redor do fruto, em virtude da redução do déficit de pressão de vapor no interior da embalagem, diminuindo a perda de água por transpiração e, consequentemente, o murchamento, o que conserva a aparência e o frescor do produto. Embora muitos filmes plásticos com diferentes permeabilidades aos gases sejam empregados, o polietileno de baixa densidade (PEBD) e o policloreto de vinila (PVC) são os mais utilizados para frutos (MOTA et al., 2006; MACHADO et al., 2007).

Assim, o presente trabalho teve como objetivo avaliar a indução da injúria por frio e o efeito da embalagem e da temperatura sobre a conservação pós-colheita de frutos de E. dysenterica.

\section{MATERIAL E MÉTODOS}

Os frutos de E. dysenterica foram colhidos no estádio verde-maduro, ainda ligados à plantamãe, na Serra da Bandeira, localizada no município de Barreiras, região oeste do Estado da Bahia, coordenadas $12^{\circ} 06^{\prime} \mathrm{S}$ e $44^{\circ} 59^{\prime} \mathrm{W}$, a uma altitude de, aproximadamente, $710 \mathrm{~m}$ acima do nível do mar.

Após a obtenção, os frutos foram transportados em balde, para o Laboratório de Botânica da Universidade Federal da Bahia, onde foram selecionados de acordo com o grau de maturação, sanidade e ausência de danos mecânicos. Posteriormente, foram lavados em água corrente, secos à temperatura ambiente e pesados em balança semianalítica para serem submetidos aos tratamentos pós-colheita.

Para isto, os frutos foram distribuídos em bandejas de poliestireno expandido, cobertas por filme PVC perfurado e sem perfuração, e bandejas sem revestimento de PVC de 10 micras, sendo que o acondicionamento foi realizado em duas temperaturas 5 (refrigeração) e $25^{\circ} \mathrm{C}$, sem controle de umidade. Nas embalagens, realizaram-se duas perfurações $(1 \mathrm{~cm})$ em cada extremidade e duas no centro. A perfuração foi realizada visando a maior circulação de ar dentro das embalagens.

Os tratamentos foram distribuídos da seguinte forma: $\mathrm{T} 1-5^{\circ} \mathrm{C}$ sem revestimento de PVC; T2 $-25^{\circ} \mathrm{C}$ sem revestimento de $\mathrm{PVC} ; \mathrm{T} 3-5^{\circ} \mathrm{C}$ com revestimento de $\mathrm{PVC}$ sem perfurações; $\mathrm{T} 4-25^{\circ} \mathrm{C}$ com revestimento de PVC sem perfurações; T5 $5^{\circ} \mathrm{C}$ com revestimento de PVC com perfurações; T6 $-25^{\circ} \mathrm{C}$ com revestimento de $\mathrm{PVC}$ com perfurações.

Durante o armazenamento, foi avaliada a durabilidade dos frutos por meio de análises diárias, 
considerando:

- pH, determinado por meio de leitura em pHmetro digital (Digimed ${ }^{\circledR}$ ) calibrado;

- medida de altura e diâmetro, por meio do paquímetro digital (King Tools ${ }^{\circledR}$ );

- perda de massa, determinada por meio da pesagem em balança semianalítica $\left(\mathrm{Bel}^{\circledR}\right)$, dos frutos, e estimada em relação à massa inicial dos frutos antes do tratamento, a partir da seguinte equação preestabelecida: $\mathrm{PMF}=\underline{(\mathrm{MFI}-\mathrm{MFF}) \times 100}$, em que, MFI,

PMF, perda de massa fresca (\%); MFI, massa fresca inicial (g); MFF, massa fresca final (g), e os dados foram transformados em perda de massa acumulada (\%) (ÁLVARES, 2006);

- coloração e firmeza, analisadas subjetivamente, a partir de uma escala-padrão para a avaliação dos frutos. Para coloração, utilizou-se de uma escala de zero a quatro, a partir do totalmente verde ao totalmente amarelado ( 0 - totalmente verde; $1-25 \%$ amarelo; $2-50 \%$ amarelo; $3-75 \%$ amarelo; 4 - totalmente amarelo). A firmeza foi inferida, a partir da percepção pelo tato, com base em uma escala de zero a dois ( 0 - totalmente firme; 1 - mediamente firme, e 2 - sem firmeza).

As amostras mantidas sob refrigeração também foram verificadas quanto à injúria por frio, considerando os critérios aparecimento de necrose e coloração marrom, sintomas característicos de injúria por frio.

O metabolismo de carboidrato também foi monitorado, a partir da quantificação de açúcar solúvel total (ASTs), açúcar redutor (AR) e açúcar não redutor (ANR). Para a quantificação dos AST, foi utilizado o método fenol sulfúrico (DUBOIS et al., 1956), sempre em duplicata. A quantificação do teor de AR foi realizada pelo método de Somogy-Nelson (NELSON, 1944). O teor de ANR foi estimado subtraindo-se o teor de açúcares redutores do teor de açúcares solúveis totais.

As avaliações foram realizadas durante todo o período de durabilidade dos frutos (5 dias), o que correspondeu à sua vida de prateleira. $\mathrm{O}$ experimento foi concluído quando os frutos apresentaram sinais de senescência, ou seja, $50 \%$ do fruto danificado, o que inviabiliza a comercialização.

O experimento foi montado em delineamento em blocos ao acaso, com três repetições, sendo a unidade experimental constituída por 6 frutos. Os dados foram submetidos à análise de variância; e as médias, comparadas pelo teste de Scott-Knott, ao nível de $5 \%$ de probabilidade, utilizando o programa estatístico Assistat, versão 7.7 beta (SILVA; AZEVEDO, 2009).

\section{RESULTADOS E DISCUSSÃO}

Os frutos de E. dysenterica tiveram durabilidade de 5 dias (dados não mostrados), independentemente do tratamento, quando apresentaram os sinais de senescência, incluindo perdas das propriedades organolépticas de textura, aroma e coloração, degenerescência dos tecidos e consequente envelhecimento e murchamento. Os tratamentos propostos aumentaram em dois dias a vida de prateleira do fruto, se comparado ao armazenamento a $28^{\circ} \mathrm{C}$ sem uso de embalagem (CALBO et al., 1990). Esta baixa vida de prateleira de frutos de E. dysenterica pode estar associada ao comportamento climatérico e à alta sensibilidade ao etileno, o que proporciona rápida evolução da coloração da epiderme e perda de firmeza da polpa (CALBO et al., 1990).

Em relação à ocorrência de injúria por frio, constatou-se que os frutos expostos à refrigeração $\left(5^{\circ} \mathrm{C}\right)$, independentemente do tipo de embalagem, apresentaram manchas marrons, indicando sensibilidade à baixa temperatura. Isto aconteceu, pois alguns produtos hortícolas provenientes de regiões tropicais e subtropicais sofrem injúria por frio, se forem armazenados abaixo da temperatura crítica determinada, apresentando sintomas como colapso e necrose de tecidos, escurecimento (coloração marrom) do fruto, suscetibilidade ao ataque por fungos e fim da vida pós-colheita (FINGER et al., 2008; DAMODARAN et al., 2010). Comportamento semelhante ao do presente trabalho foi observado em goiaba (Psidium guajava L.), também pertecente à família Myrtaceae, a qual se mostrou sensível ao dano pelo frio, não sendo indicada temperatura de armazenamento para este fruto, inferior a $5^{\circ} \mathrm{C}$ (CHITARRA; CHITARRA, 2005).

Durante a vida de prateleira, houve alteração na cor dos frutos de cagaita, de verde para amarelo, sendo que a intensidade foi dependente do tratamento (Tabela 1). Ao final da vida de prateleira, os frutos mantidos em baixa temperatura apresentaram apenas $25 \%$ da superfície amarelada, enquanto nos mantidos a $25^{\circ} \mathrm{C}$, observou-se maior alteração na coloração, visto que os frutos estavam totalmente amarelados, independentemente do uso de embalagem. A coloração é um atributo físico (visual), podendo apresentar mudanças (durante o fim) ao longo da maturação e início do amadurecimento, principalmente na casca, sendo resultado da degradação de clorofila, síntese ou revelação de pigmentos, como carotenoides e antocianinas (TUCKER,1993).

Com relação à firmeza, observou-se amolecimento apenas nos frutos submetidos ao 
ambiente climatizado $\left(25^{\circ} \mathrm{C}\right)$, pois apresentaram-se mediamente firmes, enquanto os frutos armazenados a $5^{\circ} \mathrm{C}$ se mantiveram firmes durante todo o período do experimento (Tabela 2). Este fato também foi observado por Kohatsu et al. (2011), em fruto de cajá-manga (Spondias dulcis), onde esse processo foi mais rápido quando os frutos foram mantidos a $25^{\circ} \mathrm{C}$, do que nos tratamentos sob refrigeração.

Após a alteração da cor, o amolecimento do fruto representa a mudança mais importante que ocorre no processo de maturação (AWAD, 1993). Os dados obtidos podem estar relacionados ao fato de a perda de firmeza ser acelerada pelo aumento da temperatura de armazenamento, o que promove incremento na atividade de enzimas, como celulase, pectinametilesterase (PME) e poligalacturonase (PG), as quais são responsáveis pela degradação de pectinas, celulose e hemicelulose, principais classes de polissacarídeos presentes na parede celular (SILVA et al., 2000; PRASSANA et al., 2007). Este fato foi verificado em espécies da família Myrtaceae, incluindo a goiaba (Psidium guajava L.), uma vez que, durante o amadurecimento, ocorreu aumento da atividade de enzimas hidrolíticas como a PG e PME (JAIN et al., 2001), promovendo redução da firmeza. Outro atributo a ser considerado na póscolheita é a acidez. A acidez dos frutos deve-se à presença de ácidos orgânicos, sendo que o acúmulo destes promove o aumento de sabor ácido ou azedo, pois este está relacionado com a fermentação e, neste caso, o fruto já pode estar no início da senescência, aumentando a acidez (DAMODARAN et al., 2010) devido à utilização desses compostos na respiração e sua conversão em açúcares (KOBLITZ, 2011).

Os frutos de E. dysenterica demonstraram $\mathrm{pH}$ ácido, durante todo o período de armazenamento, principalmente aqueles acondicionados sob refrigeração, cujo pH médio foi de 4,18, independentemente do tipo de embalagem (Tabela 3). Este valor está acima do encontrado por Camilo (2012) para frutos de cagaiteira coletados em Goiânia - Goiás, já que estes apresentaram pH próximo de 2,73. O aumento do $\mathrm{pH}$ está diretamente relacionado com o decréscimo da acidez ocorrido com o avanço da maturação dos frutos (NOGUEIRA, et al., 2002), aumentando, assim, a aceitação do produto.

No decorrer do período de armazenamento, verificou-se perda significativa de massa fresca dos frutos (Tabela 4), sendo esta uma característica que interfere na aceitabilidade do produto pelo consumidor, pois causa prejuízos à qualidade, principalmente pelas alterações na textura (VICENTINI et al., 1999; BRUNINI et al., 2004). Observou-se que a perda de massa foi mais intensa nos frutos mantidos sob refrigeração com revestimento de PVC sem perfuração, visto que alcançou mais de $40 \%$ de perda, e menos severa nos que foram armazenados em $25^{\circ} \mathrm{C}$ com PVC sem perfurações $(19,2 \%)$. Para os frutos mantidos sob refrigeração, independentemente da embalagem, a maior redução na massa aconteceu no $1^{\circ}$ dia, enquanto nos frutos armazenados em ambiente climatizado isto aconteceu no segundo dia. Segundo Silva et al. (2009), a perda de massa é um sintoma inicial de perda de água e pode ser atribuída, principalmente, à perda de umidade e de material de reserva, pela transpiração e respiração, respectivamente, sendo um dos principais fatores limitantes da vida útil pós-colheita dos frutos (MENEZES et al., 1995). De acordo com Chitarra e Chitarra (2005), a perda de massa máxima varia entre 5 e $10 \%$. Levando em consideração este parâmetro, os frutos de cagaita do presente estudo teriam durabilidade de dois dias. Entretanto, algumas características, tais como coloração e firmeza, mantiveram-se de acordo com as exigências do consumidor, não afetando a aparência e garantindo a durabilidade média de 5 dias.

A redução observada na massa fresca de frutos de cagaita pôde ser comprovada por alterações na altura e no diâmetro de fruto (Tabelas 5 e 6 , respectivamente). A altura dos frutos diferiu significativamente em função dos tratamentos utilizados, após 1; 2 e 5 dias de armazenamento (Tabela 5). Os frutos mantidos sob refrigeração demonstraram a mesma redução de altura $(8,5 \%)$ durante a vida de prateleira, independentemente da embalagem, sendo inferior à diminuição desta característica em frutos armazenados em ambiente climatizado (12\%, em média). A altura inicial dos frutos de cagaita foi de $27,1 \mathrm{~mm}$, medida inferior à registrada por Camilo (2012), o qual encontrou valores em torno de $36,6 \mathrm{~mm}$. Isto pode ter acontecido devido às condições edafoclimáticas, já que foram coletadas em ambientes diferentes.

A partir da análise da variação do diâmetro, percebeu-se que os frutos de cagaita não mostraram diferença significativa ao longo do armazenamento, independentemente do tratamento utilizado. Ao analisar os dias, constatou-se que, assim como na altura, os frutos mantidos sob refrigeração demonstraram menores reduções de diâmetro, $6 \%$ em média, e os frutos mantidos em ambiente climatizado mostraram maiores reduções, sendo que a $25^{\circ} \mathrm{C}$ com PVC a diminuição foi maior, $12 \%$ (Tabela 6). O diâmetro médio inicial dos frutos foi $29,5 \mathrm{~mm}$ menor do que o encontrado por Camilo (2012), que foi de $38,3 \mathrm{~mm}$.

Quanto ao metabolismo de carboidratos, 
percebeu-se redução significativa na quantidade de açúcar solúvel total (AST) ao longo dos dias (Tabela 7). Entretanto, entre o $4^{\circ}$ e o $5^{\circ}$ dia de armazenamento, verificou-se aumento na quantidade de AST em frutos mantidos sob refrigeração $\left(5^{\circ} \mathrm{C}\right)$ com e sem revestimento de PVC. Tal comportamento também foi constatado por Santos et al. (2006) em pitangas (Eugenia uniflora), pois verificouse uma tendência de aumento nos conteúdos de AST nos frutos mantidos a $10^{\circ} \mathrm{C}$, o que pode estar relacionado à perda de água sofrida dos frutos. Nos frutos de cagaita, verificou-se, também, redução de $\mathrm{pH}$. Diante do menor conteúdo de água nos frutos, houve concentração de açúcares e ácidos, elevando os níveis de AST e diminuindo o $\mathrm{pH}$, o que ocorre devido à grande demanda energética do sistema para a continuação dos processos metabólicos, com a hidrólise de carboidratos de cadeia longa e consequente aumento nos teores de sacarose, frutose e glicose (OLIVEIRA et al., 2001). O aumento do teor de açúcares solúveis totais pode estar relacionado, também, à conversão de polissacarídeos da parede celular em açúcares solúveis (CHITARRA; CHITARRA, 2005).

Avaliando-se o teor de açúcar redutor (AR), verificou-se redução significativa no conteúdo ao longo da vida de prateleira dos frutos de $E$. dysenterica, sendo que a maior redução aconteceu nos frutos armazenados a $25^{\circ} \mathrm{C}$ sem $\mathrm{PVC}$, diminuindo em quase $80 \%$, e a menor aconteceu nos frutos submetidos a $5^{\circ} \mathrm{C}$ com PVC sem perfurações, com $36 \%$ (Tabela 8). Este resultado pode ser devido à parte da sacarose ter sido hidrolisada para que os monossacarídeos (glicose e frutose) fossem metabolizados, gerando energia aos processos metabólicos (OLIVEIRA et al., 2001). Observou-se que o conteúdo de AR nos frutos armazenados sob refrigeração foi superior aos tratamentos em ambiente climatizado $\left(25^{\circ} \mathrm{C}\right)$. Nos frutos armazenados sob temperatura de $25^{\circ} \mathrm{C}$, percebeu-se perda de firmeza e mudança de coloração, o que se deve, provavelmente, ao aumento na atividade respiratória quando os frutos são mantidos em temperaturas mais altas, proporcionando o consumo de reservas (LIMA; DURIGAN, 2000). Os resultados obtidos corroboram os encontrados por Pereira et al. (2005) em goiabas (Psidium guajava L.) armazenadas sob refrigeração com e sem embalagem, pois tanto o teor de glicose quanto o de frutose apresentaram redução constante desde o primeiro até o último dia de armazenamento, tanto para frutos embalados como para os não embalados.

Para o açúcar não redutor (ANR), verificouse diferença significativa nos valores encontrados, sendo que a maior redução ocorreu nos frutos acondicionados a $5^{\circ} \mathrm{C}$ com revestimento de $\mathrm{PVC}$, independentemente da perfuração, com decréscimo médio de quase $90 \%$ na quantidade de ANR (Tabela 9). Diferentemente do padrão geral observado, no $5^{\circ}$ dia de armazenamento, pôde-se perceber, em todos os tratamentos utilizados neste estudo, que houve aumento no conteúdo de ANR no $5^{\circ}$ dia do armazenamento. Fato semelhante foi observado em trabalho com pêssego (Prunus persica L. Bastsch), pois verificou-se que os valores de sacarose aumentaram durante $o$ armazenamento (OLIVEIRA et al., 2001). Isto aconteceu, provavelmente, porque os carboidratos mudam tanto em qualidade como em quantidade durante o amadurecimento do fruto (CORRÊA et al., 2000). Em geral, os frutos contêm maior quantidade de açúcares redutores do que de sacarose (DAMODARAN et al., 2010), comportamento verificado no presente trabalho. 
TABELA 1- Valores médios de coloração em frutos de Eugenia dysenterica armazenados sob diferentes temperaturas e embalagens.

\begin{tabular}{ccccccc}
\hline Tratamento & Dia 0 & Dia 1 & Dia 2 & Dia 3 & Dia 4 & Dia 5 \\
\hline $\mathbf{5}^{\circ} \mathbf{C}$ sem PVC & 0 & 0 & 1 & 1 & 1 & 1 \\
$\mathbf{2 5}^{\circ} \mathbf{C}$ sem PVC & 0 & 0 & 1 & 1 & 3 & 4 \\
$\mathbf{5}^{\circ} \mathrm{C}$ com PVC sem perfurações & 0 & 0 & 1 & 1 & 1 & 1 \\
$\mathbf{2 5}^{\circ} \mathrm{C}$ com PVC sem perfurações & 0 & 0 & 1 & 2 & 3 & 4 \\
$\mathbf{5}^{\circ} \mathbf{C}$ com PVC com perfurações & 0 & 0 & 1 & 1 & 1 & 1 \\
$\mathbf{2 5}^{\circ} \mathrm{C}$ com PVC com perfurações & 0 & 0 & 1 & 1 & 3 & 4 \\
\hline
\end{tabular}

0 - totalmente verde; 1 - 25\% amarelo; 2 - 50\% amarelo; 3 - 75\% amarelo; 4 - totalmente amarelo.

TABELA 2 -Valores médios de firmeza em frutos de Eugenia dysenterica armazenados sob diferentes temperaturas e embalagens.

\begin{tabular}{ccccccc}
\hline Tratamento & Dia 0 & Dia 1 & Dia 2 & Dia 3 & Dia 4 & Dia 5 \\
\hline $\mathbf{5}^{\circ} \mathbf{C}$ sem PVC & 0 & 0 & 0 & 0 & 0 & 0 \\
$\mathbf{2 5}^{\circ} \mathbf{C}$ sem PVC & 0 & 0 & 0 & 0 & 1 & 1 \\
$\mathbf{5}^{\circ} \mathbf{C}$ com PVC sem perfurações & 0 & 0 & 0 & 0 & 0 & 0 \\
$\mathbf{2 5}^{\circ} \mathbf{C}$ com PVC sem perfurações & 0 & 0 & 0 & 1 & 1 & 1 \\
$\mathbf{5}^{\circ} \mathbf{C}$ com PVC com perfurações & 0 & 0 & 0 & 0 & 0 & 0 \\
$\mathbf{2 5}^{\circ} \mathbf{C}$ com PVC com perfurações & 0 & 0 & 0 & 0 & 1 & 1 \\
\hline
\end{tabular}

0 - totalmente firme; 1 - mediamente firme; 2 - sem firmeza.

TABELA 3- Valores médios de $\mathrm{pH}$ em frutos de Eugenia dysenterica armazenados sob diferentes temperaturas e embalagens.

\begin{tabular}{ccccccc}
\hline Tratamento & Dia 0 & Dia 1 & Dia 2 & Dia 3 & Dia 4 & Dia 5 \\
\hline $\mathbf{5}^{\circ}$ C sem PVC & $4,20 \mathrm{aA}$ & $4,18 \mathrm{aA}$ & $4,25 \mathrm{aA}$ & $4,15 \mathrm{aA}$ & $4,16 \mathrm{aA}$ & $4,07 \mathrm{bA}$ \\
$\mathbf{2 5}^{\circ} \mathrm{C}$ sem PVC & $4,20 \mathrm{aA}$ & $4,30 \mathrm{aA}$ & $3,92 \mathrm{aA}$ & $4,21 \mathrm{aA}$ & $4,36 \mathrm{aA}$ & $4,37 \mathrm{aA}$ \\
$\mathbf{5}^{\circ} \mathrm{C}$ com PVC sem perfurações & $4,20 \mathrm{aA}$ & $4,09 \mathrm{aB}$ & $4,26 \mathrm{aA}$ & $4,22 \mathrm{aA}$ & $4,18 \mathrm{aA}$ & $4,14 \mathrm{bB}$ \\
$\mathbf{2 5}^{\circ} \mathrm{C}$ com PVC sem perfurações & $4,20 \mathrm{aA}$ & $4,21 \mathrm{aA}$ & $4,26 \mathrm{aA}$ & $4,47 \mathrm{aA}$ & $4,39 \mathrm{aA}$ & $4,56 \mathrm{aA}$ \\
$\mathbf{5}^{\circ} \mathbf{C}$ com PVC com perfurações & $4,20 \mathrm{aA}$ & $4,21 \mathrm{aA}$ & $4,32 \mathrm{aA}$ & $4,18 \mathrm{aA}$ & $4,04 \mathrm{aA}$ & $4,10 \mathrm{bA}$ \\
$\mathbf{2 5}^{\circ} \mathrm{C}$ com PVC com perfurações & $4,20 \mathrm{aA}$ & $4,21 \mathrm{aA}$ & $4,21 \mathrm{aA}$ & $4,27 \mathrm{aA}$ & $4,24 \mathrm{aA}$ & $4,46 \mathrm{aA}$ \\
\hline
\end{tabular}

Médias seguidas pela mesma letra, minúscula na coluna e maiúscula na linha, não diferem entre si, pelo teste de Scott-Knott, ao nível de $5 \%$ de probabilidade.

TABELA 4- Valores médios de perda de massa (\%) em frutos de Eugenia dysenterica armazenados sob diferentes temperaturas e embalagens.

\begin{tabular}{ccrcccc}
\hline Tratamento & Dia 0 & Dia 1 & Dia 2 & Dia 3 & Dia 4 & Dia 5 \\
\hline $\mathbf{5}^{\circ} \mathbf{C}$ sem PVC & $0,0 \mathrm{aF}$ & $7,6 \mathrm{dE}$ & $12,8 \mathrm{eD}$ & $20,2 \mathrm{cC}$ & $21,1 \mathrm{eB}$ & $21,3 \mathrm{eA}$ \\
$\mathbf{2 5}^{\circ} \mathbf{C}$ sem PVC & $0,0 \mathrm{aF}$ & $6,6 \mathrm{eE}$ & $17,5 \mathrm{cD}$ & $19,0 \mathrm{eC}$ & $23,8 \mathrm{~dB}$ & $26,7 \mathrm{dA}$ \\
$\mathbf{5}^{\mathbf{C}} \mathrm{C}$ com PVC sem perfurações & $0,0 \mathrm{aF}$ & $19,2 \mathrm{aE}$ & $25,9 \mathrm{aD}$ & $37,7 \mathrm{aC}$ & $38,1 \mathrm{aB}$ & $43,0 \mathrm{aA}$ \\
$\mathbf{2 5}^{\circ} \mathbf{C}$ com PVC sem perfurações & $0,0 \mathrm{aF}$ & $5,5 \mathrm{fE}$ & $11,9 \mathrm{fD}$ & $12,0 \mathrm{fC}$ & $12,6 \mathrm{fB}$ & $19,2 \mathrm{fA}$ \\
$\mathbf{5}^{\circ} \mathbf{C}$ com PVC com perfurações & $0,0 \mathrm{aF}$ & $11,5 \mathrm{bE}$ & $15,3 \mathrm{dD}$ & $20,1 \mathrm{dC}$ & $27,2 \mathrm{cB}$ & $27,7 \mathrm{cA}$ \\
$\mathbf{2 5}^{\circ} \mathbf{C}$ com PVC com perfurações & $0,0 \mathrm{aF}$ & $9,9 \mathrm{cE}$ & $23,9 \mathrm{bD}$ & $31,0 \mathrm{bC}$ & $35,8 \mathrm{bB}$ & $38,9 \mathrm{bA}$ \\
\hline
\end{tabular}

Médias seguidas pela mesma letra, minúscula na coluna e maiúscula na linha, não diferem entre si, pelo teste de Scott-Knott, ao nível de $5 \%$ de probabilidade. 
TABELA 5- Valores médios de altura ( $\mathrm{mm})$ de frutos de Eugenia dysenterica armazenados sob diferentes temperaturas e embalagens.

\begin{tabular}{ccccccc}
\hline Tratamento & Dia 0 & Dia 1 & Dia $\mathbf{2}$ & Dia $\mathbf{3}$ & Dia 4 & Dia 5 \\
\hline $\mathbf{5}^{\circ} \mathbf{C}$ sem PVC & $27,1 \mathrm{aA}$ & $26,3 \mathrm{bA}$ & $25,3 \mathrm{bB}$ & $24,9 \mathrm{aB}$ & $24,8 \mathrm{aB}$ & $24,8 \mathrm{aB}$ \\
$\mathbf{2 5}^{\circ} \mathbf{C}$ sem PVC & $27,1 \mathrm{aA}$ & $26,2 \mathrm{bB}$ & $25,2 \mathrm{bD}$ & $25,6 \mathrm{aC}$ & $25,1 \mathrm{aD}$ & $24,2 \mathrm{bE}$ \\
$\mathbf{5}^{\circ} \mathbf{C}$ com PVC sem perfurações & $27,1 \mathrm{aA}$ & $25,7 \mathrm{bB}$ & $25,3 \mathrm{bB}$ & $25,2 \mathrm{aB}$ & $24,8 \mathrm{aB}$ & $24,8 \mathrm{aB}$ \\
$\mathbf{2 5}^{\circ} \mathbf{C}$ com PVC sem perfurações & $27,1 \mathrm{aA}$ & $26,0 \mathrm{bB}$ & $25,7 \mathrm{bB}$ & $25,0 \mathrm{aB}$ & $23,5 \mathrm{aC}$ & $23,0 \mathrm{cC}$ \\
$\mathbf{5}^{\circ} \mathbf{C}$ com PVC com perfurações & $27,1 \mathrm{aA}$ & $28,5 \mathrm{aA}$ & $27,7 \mathrm{aA}$ & $27,0 \mathrm{aA}$ & $26,0 \mathrm{aA}$ & $24,8 \mathrm{aA}$ \\
$\mathbf{2 5}^{\circ} \mathbf{C}$ com PVC com perfurações & $27,1 \mathrm{aA}$ & $26,0 \mathrm{bB}$ & $25,6 \mathrm{bB}$ & $25,6 \mathrm{aB}$ & $24,8 \mathrm{aC}$ & $24,0 \mathrm{bC}$ \\
\hline
\end{tabular}

Médias seguidas pela mesma letra, minúscula na coluna e maiúscula na linha, não diferem entre si, pelo teste de Scott-Knott, ao nível de $5 \%$ de probabilidade.

TABELA 6- Valores médios de diâmetro ( $\mathrm{mm}$ ) de frutos de Eugenia dysenterica armazenados sob diferentes temperaturas e embalagens.

\begin{tabular}{ccccccc}
\hline Tratamento & Dia 0 & Dia 1 & Dia $\mathbf{2}$ & Dia 3 & Dia 4 & Dia 5 \\
\hline $\mathbf{5}^{\circ} \mathbf{C}$ sem PVC & $29,5 \mathrm{aA}$ & $29,3 \mathrm{aA}$ & $28,8 \mathrm{aA}$ & $27,6 \mathrm{aB}$ & $27,8 \mathrm{aB}$ & $27,6 \mathrm{aB}$ \\
$\mathbf{2 5}^{\circ} \mathbf{C}$ sem PVC & $29,5 \mathrm{aA}$ & $27,3 \mathrm{aB}$ & $27,8 \mathrm{aB}$ & $27,5 \mathrm{aB}$ & $27,2 \mathrm{aB}$ & $27,1 \mathrm{aB}$ \\
$\mathbf{5}^{\circ} \mathbf{C}$ com PVC sem perfurações & $29,5 \mathrm{aA}$ & $28,7 \mathrm{aB}$ & $28,4 \mathrm{aC}$ & $28,0 \mathrm{aC}$ & $27,8 \mathrm{aC}$ & $27,6 \mathrm{aC}$ \\
$\mathbf{2 5}^{\circ} \mathbf{C}$ com PVC sem perfurações & $29,5 \mathrm{aA}$ & $28,7 \mathrm{aA}$ & $28,3 \mathrm{aA}$ & $28,3 \mathrm{aA}$ & $27,4 \mathrm{aA}$ & $26,0 \mathrm{aA}$ \\
$\mathbf{5}^{\circ} \mathbf{C}$ com PVC com perfurações & $29,5 \mathrm{aA}$ & $29,3 \mathrm{aA}$ & $29,8 \mathrm{aA}$ & $29,2 \mathrm{aA}$ & $27,9 \mathrm{aB}$ & $27,9 \mathrm{aB}$ \\
$\mathbf{2 5}^{\circ} \mathbf{C}$ com PVC com perfurações & $29,5 \mathrm{aA}$ & $28,8 \mathrm{aA}$ & $28,1 \mathrm{aA}$ & $27,1 \mathrm{aA}$ & $27,0 \mathrm{aA}$ & $26,8 \mathrm{aA}$ \\
\hline
\end{tabular}

Médias seguidas pela mesma letra, minúscula na coluna e maiúscula na linha, não diferem entre si, pelo teste de Scott-Knott, ao nível de $5 \%$ de probabilidade.

TABELA 7- Valores médios de açúcar solúvel total (\%) em frutos de Eugenia dysenterica armazenados sob diferentes temperaturas e embalagens.

\begin{tabular}{ccccccc}
\hline Tratamento & Dia 0 & Dia 1 & Dia 2 & Dia 3 & Dia 4 & Dia 5 \\
\hline $\mathbf{5}^{\circ} \mathbf{C}$ sem PVC & $11,6 \mathrm{aA}$ & $8,1 \mathrm{bB}$ & $6,0 \mathrm{cC}$ & $5,6 \mathrm{bC}$ & $3,9 \mathrm{bD}$ & $4,6 \mathrm{aD}$ \\
$\mathbf{2 5}^{\circ} \mathbf{C}$ sem PVC & $11,6 \mathrm{aA}$ & $10,6 \mathrm{aA}$ & $7,3 \mathrm{bB}$ & $4,6 \mathrm{dC}$ & $4,6 \mathrm{aC}$ & $3,5 \mathrm{aC}$ \\
$\mathbf{5}^{\circ} \mathbf{C}$ com PVC sem perfurações & $11,6 \mathrm{aA}$ & $11,5 \mathrm{aA}$ & $6,7 \mathrm{cB}$ & $6,7 \mathrm{aB}$ & $4,2 \mathrm{bD}$ & $4,9 \mathrm{aC}$ \\
$\mathbf{2 5}^{\circ} \mathbf{C}$ com PVC sem perfurações & $11,6 \mathrm{aA}$ & $9,3 \mathrm{aB}$ & $5,8 \mathrm{cC}$ & $5,8 \mathrm{bC}$ & $5,1 \mathrm{aC}$ & $4,1 \mathrm{aD}$ \\
$\mathbf{5}^{\circ} \mathbf{C}$ com PVC com perfurações & $11,6 \mathrm{aA}$ & $8,1 \mathrm{bB}$ & $8,6 \mathrm{aB}$ & $6,0 \mathrm{bC}$ & $4,2 \mathrm{bD}$ & $3,7 \mathrm{aD}$ \\
$\mathbf{2 5}^{\circ} \mathbf{C}$ com PVC com perfurações & $11,6 \mathrm{aA}$ & $5,9 \mathrm{cB}$ & $5,6 \mathrm{cB}$ & $5,3 \mathrm{cB}$ & $4,2 \mathrm{bC}$ & $3,9 \mathrm{aC}$ \\
\hline
\end{tabular}

Médias seguidas pela mesma letra, minúscula na coluna e maiúscula na linha, não diferem entre si, pelo teste de Scott-Knott, ao nível de $5 \%$ de probabilidade.

TABELA 8 -Valores médios de açúcar redutor (\%) em frutos de Eugenia dysenterica armazenados sob diferentes temperaturas e embalagens.

\begin{tabular}{ccccccc}
\hline Tratamento & Dia 0 & Dia 1 & Dia $\mathbf{2}$ & Dia $\mathbf{3}$ & Dia 4 & Dia 5 \\
\hline $\mathbf{5}^{\circ} \mathbf{C}$ sem PVC & $6,9 \mathrm{aA}$ & $5,9 \mathrm{aB}$ & $4,9 \mathrm{bC}$ & $4,3 \mathrm{bC}$ & $3,5 \mathrm{aD}$ & $2,8 \mathrm{bD}$ \\
$\mathbf{2 5}^{\circ} \mathbf{C}$ sem PVC & $6,9 \mathrm{aA}$ & $6,9 \mathrm{aA}$ & $5,1 \mathrm{bB}$ & $4,1 \mathrm{bC}$ & $3,0 \mathrm{aD}$ & $1,4 \mathrm{cE}$ \\
$\mathbf{5}^{\circ} \mathbf{C}$ com PVC sem perfurações & $6,9 \mathrm{aA}$ & $6,6 \mathrm{aA}$ & $4,8 \mathrm{bB}$ & $5,3 \mathrm{aB}$ & $5,0 \mathrm{aB}$ & $4,4 \mathrm{aB}$ \\
$\mathbf{2 5}^{\circ} \mathbf{C}$ com PVC sem perfurações & $6,9 \mathrm{aA}$ & $6,5 \mathrm{aA}$ & $5,2 \mathrm{bB}$ & $4,5 \mathrm{bC}$ & $4,3 \mathrm{aC}$ & $2,2 \mathrm{cD}$ \\
$\mathbf{5}^{\circ} \mathbf{C}$ com PVC com perfurações & $6,9 \mathrm{aA}$ & $6,1 \mathrm{aA}$ & $5,7 \mathrm{aA}$ & $5,2 \mathrm{aA}$ & $3,7 \mathrm{aB}$ & $2,7 \mathrm{bB}$ \\
$\mathbf{2 5}^{\circ} \mathbf{C}$ com PVC com perfurações & $6,9 \mathrm{aA}$ & $4,8 \mathrm{aB}$ & $4,8 \mathrm{bB}$ & $4,1 \mathrm{bC}$ & $3,8 \mathrm{aC}$ & $2,1 \mathrm{cD}$ \\
\hline
\end{tabular}

Médias seguidas pela mesma letra, minúscula na coluna e maiúscula na linha, não diferem entre si, pelo teste de Scott-Knott, ao nível de $5 \%$ de probabilidade. 
TABELA 9- Valores médios de açúcar não-redutor (\%) em frutos de Eugenia dysenterica armazenados sob diferentes temperaturas e embalagens.

\begin{tabular}{ccccccc}
\hline Tratamento & Dia 0 & Dia 1 & Dia $\mathbf{2}$ & Dia $\mathbf{3}$ & Dia 4 & Dia $\mathbf{5}$ \\
\hline $\mathbf{5}^{\circ} \mathbf{C}$ sem PVC & $4,7 \mathrm{aA}$ & $2,2 \mathrm{aB}$ & $1,1 \mathrm{bB}$ & $1,3 \mathrm{aB}$ & $0,4 \mathrm{aB}$ & $1,8 \mathrm{aB}$ \\
$\mathbf{2 5}^{\circ} \mathbf{C}$ sem PVC & $4,7 \mathrm{aA}$ & $3,6 \mathrm{aA}$ & $2,3 \mathrm{aB}$ & $0,5 \mathrm{aB}$ & $1,5 \mathrm{aB}$ & $2,1 \mathrm{aB}$ \\
$\mathbf{5}^{\circ} \mathbf{C}$ com PVC sem perfurações & $4,7 \mathrm{aA}$ & $4,8 \mathrm{aA}$ & $1,8 \mathrm{aB}$ & $1,4 \mathrm{aB}$ & $0,2 \mathrm{aB}$ & $0,5 \mathrm{bB}$ \\
$\mathbf{2 5}^{\circ} \mathbf{C}$ com PVC sem perfurações & $4,7 \mathrm{aA}$ & $2,8 \mathrm{aA}$ & $0,6 \mathrm{bB}$ & $1,3 \mathrm{aB}$ & $0,9 \mathrm{aB}$ & $1,9 \mathrm{aB}$ \\
$\mathbf{5}^{\circ} \mathbf{C}$ com PVC com perfurações & $4,7 \mathrm{aA}$ & $2,0 \mathrm{aC}$ & $2,9 \mathrm{aB}$ & $0,8 \mathrm{aC}$ & $0,5 \mathrm{aC}$ & $1,0 \mathrm{bC}$ \\
$\mathbf{2 5}^{\circ} \mathbf{C}$ com PVC com perfurações & $4,7 \mathrm{aA}$ & $1,0 \mathrm{aB}$ & $0,8 \mathrm{bB}$ & $1,2 \mathrm{aB}$ & $0,4 \mathrm{aB}$ & $1,8 \mathrm{aB}$ \\
\hline
\end{tabular}

Médias seguidas pela mesma letra, minúscula na coluna e maiúscula na linha, não diferem entre si, pelo teste de Scott-Knott, ao nível de $5 \%$ de probabilidade.

\section{CONCLUSÃO}

Os frutos de Eugenia dysenterica mostraram vida de prateleira curta (5 dias), quando estes apresentaram $50 \%$ de dano, percebido a partir da aparência. A temperatura de $5^{\circ} \mathrm{C}$ não foi eficiente para a conservação dos frutos, pois promoveu o aparecimento de sintomas de injúria por frio. Quando armazenados a $25^{\circ} \mathrm{C}$, os frutos apresentaram características climatéricas, incluindo mudança de coloração, perda de firmeza, aumento de $\mathrm{pH}$ e intenso consumo de carboidratos. O uso de embalagens, praticamente, não promoveu efeitos significativos na conservação dos frutos.

\section{AGRADECIMENTOS}

À Fundação de Amparo à Pesquisa do Estado da Bahia - FAPESB, pelo apoio financeiro.

\section{REFERÊNCIAS}

ÁlVARES, V.S. Pré-resfriamento, embalagem e hidratação pós-colheita de salsinha. 2006. 161f. Tese (Doutorado em Fitotecnia) - Universidade Federal de Viçosa,Viçosa, 2006.

ÁLVAREZ-ARMENTA, R.; SAUCEDO-VELOZ, C.; CHÁVEZ-FRANCO, S.; MEDINA-URRUTIA, V.; COLINAS-LEÓN, M.T.; BÁEZ-SAÑUDO, R. Reguladores de crecimiento en la maduración y senescencia de frutos de limón mexicano. Agricultura Técnica en México, México, v. 34, n. 1, p. 5-11, 2008.

ANTUNES, L.E.C.; FILHO, J.D.; SOUZA, C.M. Conservação pós-colheita de frutos de amoreirapreta. Pesquisa Agropecuária Brasileira, Brasília, v. 38, n. 3, p. 413-419, 2003.
AWAD, M. Fisiologia pós-colheita de frutos. São Paulo: Nobel, 1993. 114p.

BRUNINI, M.A.; OLIVEIRA, A.L.; SALANDINI, C.A.R.; BAZZO, F.R. Influência de embalagens e temperatura no armazenamento de jabuticabas (Myrciaria jabuticaba (Vell) Berg) cv 'Sabará'. Ciência e Tecnologia de Alimentos, Campinas, v. 24, n. 3, p. 378-383, 2004.

CALBO, M.E.R.; LIMA, J.N.; CALBO, A.G. Fisiologia pós-colheita de frutos de cagaita. Revista Brasileira de Fisiologia Vegetal, Brasília, v. 2, n. 1, p. 15-18, 1990.

CAMILO, Y.M.V. Seleção de plantas e caracterização de frutos de cagaiteiras (Eugenia dysenterica DC.). 2012. 96f. Dissertação (Mestrado em Agronomia) - Universidade Federal de Goiás, Escola de Agronomia e Engenharia de Alimentos, Goiânia, 2012.

CARDOSO, L.M.; MARTINO, H.S.D.; MOREIRA, A.V.B.; RIBEIRO, S.M.R.; PINHEIRO-SANT'ANA, H.M. Cagaita (Eugenia dysenterica DC.) of the Cerrado of Minas Gerais, Brazil: Physical and chemical characterization, carotenoids and vitamins. Food Research International, New York, v.44, n. 7, p. 2151-2154, 2011.

CHITARRA, M.I.F.; CHITARRA, A.B. Pós-colheita de frutos e hortaliças: fisiologia e manuseio. 2.ed. Lavras: UFLA, 2005. 783p.

CORRÊA, A.D.; ABREU, C.M.P.; SANTOS, C.D.; RIBEIRO, L.J. Constituintes químicos da frutade-lobo (Solanum lycocarpum st. hil.) durante a maturação. Ciência Agrotecnologia, Lavras, v. 24, n. 1, p. 130-135, 2000. 
DAMODARAN, S.; PARKIN, K.L.; FENNEMA, O.R. Química de alimentos de Fennema. 4.ed. Porto Alegre: Artmed, 2010. 900p.

DUBOIS, M.; GILLES, K.A.; HAMILTON, J.K.; REBERS, P.A.; SMITH, F. Colorimetric method for determination of sugars and related substances. Analytical Chemistry, Washington, v. 28, n. 3, p. 350-356, 1956.

FINGER, F.L.; DELLA-JUSTINA, M.E.; CASALI, V.W.D.; PUIATTI, M. Temperature and modified atmosphere affect the quality of okra. Scientia Agricola, Piracicaba, v. 65, n. 4, p. 360-364, 2008.

JAIN, N.; DHAWAN, K.; MALHOTRA, S.P.; SIDDIQUI, S.; SINGH, R. Compositional and enzymatic changes in guava (Psidium guajava L.) fruits during ripening. Acta Physiologiae Plantarum, Krakov, v. 23, n. 3, p. 357-362, 2001.

K O B LITZ, M.G.B. Matérias-primas alimentícias: composição e controle de qualidade. Rio de Janeiro: Guanabara Koogan, 2011. 301p.

KOHATSU, D.S.; ZUCARELI, V.; BRAMBILLA, W.P.; EVANGELISTA, R.M. Qualidade de frutos de cajá-manga armazenados sob diferentes temperaturas. Revista Brasileira de Fruticultura, Jaboticabal, v. 33, p. 344-349, 2011. Número Especial.

LIMA, M.A.; DURIGAN, J.F. Conservação de goiaba 'Pedro Sato' associando-se refrigeração com diferentes embalagens plásticas. Revista Brasileira de Fruticultura, Jaboticabal, v. 22, n. 2, p. 232-236, 2000.

MACHADO, N.P.; COUTINHO, E.F.; CAETANO, E.R. Embalagens plásticas e refrigeração na conservação pós-colheita de jabuticaba. Revista Brasileira de Fruticultura, Jaboticabal, v. 29, n.1, p. 166-168, 2007.

MENEZES, J.B.; CHITARRA, A.B.; CHITARRA, M.I.F.; CARVALHO, H.A. Caracterização póscolheita do melão amarelo "Agroflora 646". Horticultura Brasileira, Brasília, v. 13, n. 2, p. 150-153, 1995.
MOTA, W.F.; SALOMÃO, L.C.C.; NERES, C.R.L.; MIZOBUTSI, G.P.; NEVES, L.L.M. Uso de cera de carnaúba e saco plástico poliolefínico na conservação pós-colheita do maracujá-amarelo. Revista Brasileira de Fruticultura, Jaboticabal, v. 28, n. 2, p. 190-193, 2006.

NAVES, R.V.; BORGES, J.D.; CHAVES, L.J. A cagaiteira. Revista Brasileira de Fruticultura, Jaboticabal, v. 24, n. 2, 2002. Capa.

NELSON, N.A Photometric adaptation of Somogy method for determination of glucose. Journal Biology Chemistry, Baltimore, v. 135, n. 1, p. 13675, 1944.

NOGUEIRA, R.J.M.C.; MORAES, J.A.P.V.; BURITY, H.A.; SILVA JUNIOR, J.F. Efeito do estádio de maturação dos frutos nas características físico-químicas de acerola. Pesquisa Agropecuária Brasileira, Brasília, v.37, n.4, p. 463-470, 2002.

OLIVEIRA, M.A.; CEREDA, M.P.; CABELLO, C.; URBANO, L.H. Quantificação de açúcares em pêssegos da variedade Biuti, armazenados sob condições de ambiente e refrigeração. Revista Brasileira de Fruticultura, Jaboticabal, v. 23, n. 2, p. 424-427, 2001.

PEREIRA, T.; CARLOS, L.A.; OLIVEIRA, J.G.de; MONTEIRO, A.R. Características físicas e químicas de goiaba cv. cortibel (Psidium guajava) estocadas sob refrigeração em filmes $\mathrm{X}$-tend. Alimentos e Nutrição, Araraquara, v. 16, n. 1, p. 11-16, 2005.

PRASSANA, V. PRABHA, T.N.; THARANATHAN, R.N. Fruit ripening phenomena an overview. Critical Reviews in Food Science and Nutrition, Boca Raton, v. 47, n. 1, p. 1-19, 2007.

RAHMAN, M.S. Manual de conservación de los alimentos. Zaragoza: Acribia, 2003. 863p.

SAMIRA, A.; WOLDETSADIK, K.; WORKNEH, T.S. Postharvest quality and shelf life of some hot pepper varieties. Journal of Food Science and Technology, Mysore, v. 50, n. 5, p. 842-855, 2011.

SANTOS, A.F.; SILVA, S.M.; ALVES, R.E. Armazenamento de pitanga sob atmosfera modificada e refrigeração: I-transformações químicas em pós-colheita. Revista Brasileira de Fruticultura, Jaboticabal, v. 28, n. 1, p. 36-41, 2006. 
SILVA, D.F.P.; SALOMAO, L.C.C.; SIQUEIRA, D.L.; CECON, P.R.; ROCHA, A. Potassium permanganate effects in postharvest conservation of the papaya cultivar 'Sunrise Golden'. Pesquisa Agropecuária Brasileira, Brasília, v. 44, n. 7, p. 669-675, 2009.

SILVA, F.A.S.; AZEVEDO, C.A.V. Principal components analysis in the Software AssistatStatistical Attendance. In: WORLD CONGRESS ON COMPUTERS IN AGRICULTURE, 7., 2009, Reno. Proceedings... St Joseph: American Society of Agricultural and Biological Engineers, 2009.

SILVA, J.S.; FINGER, F.L.; CORRÊA, P.C. Armazenamento de frutas e hortaliças. In: SILVA, J.S. (Org.). Secagem e armazenagem de produtos agrícolas. Viçosa: Aprenda Fácil, 2000. p.469-502.

SILVA, M.R.; LACERDA, D.B.C.L.; SANTOS, G.G.; MARTINS, D.M.O. Caracterização química de frutos nativos do cerrado. Ciência Rural, Santa Maria, v. 38, n. 6, p. 1790-1793, 2008.
SILVA, R.S.M.; CHAVES, L.J.; NAVES, R.V. Caracterização de frutos e árvores de cagaita (Eugenia dysenterica DC.) no sudeste do estado de Goiás, Brasil. Revista Brasileira de Fruticultura, Jaboticabal, v. 23, n. 2, p. 330-334, 2001.

TUCKER, G.A. Introduction. In: SEYMOR, G.B.; TAYLOR, J.E.; TUCKER, G.A. Biochemistry of fruit ripening. New York: Chapman \& Hall, 1993. p. 2-51.

VICENTINI, N.M.; CASTRO, T.M.R.; CEREDA, M.P. Influência da fécula de mandioca na qualidade pós-colheita de frutos de pimentão (Capsicum annum L.). Ciência e Tecnologia de Alimentos, Campinas, v. 19, n. 1, p. 127-30, 1999. 